\title{
EXOTIC NUCLEI FROM A THEORETICAL PERSPECTIVE
}

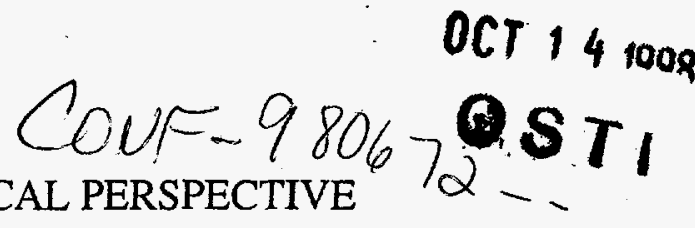

\section{W. Nazarewicz}

Department of Physics, University of Tennessee, Knoxville, Tennessee 37996

Physics Division, Oak Ridge National Laboratory, Oak Ridge, TN 37831

Institute of Theoretical Physics, University of Warsaw

ul. Hoza 69, PL-00-681 Warsaw, Poland

to be published in

Proceedings of 2nd International Conference on Exotic Nuclei and Atomic Masses (ENAM98)

Bellaire, Michigan

June 23-27, 1998

\section{NASTER}

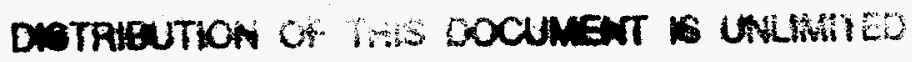

*Oak Ridge National Laboratory is managed by Lockheed Martin Energy Research Corp. for the U.S. Department of Energy under Contract No. DE-AC05-96OR22464.

"The submitted manuscript has been authored by a contractor of the U.S. Government under contract No. DE-AC05-96OR22464. Accordingly, the U.S. Government retains a nonexclusive, royalty-free license to publish or reproduce the published form of this contribution, or allow others to do so, for U.S. Government purposes." 


\section{DISCLAIMER}

This report was prepared as an account of work sponsored by an agency of the United States Government. Neither the United States Government nor any agency thereof, nor any of their employees, makes any warranty, express or implied, or assumes any legal liability or responsibility for the accuracy, completeness, or usefulness of any information, apparatus, product, or process disclosed, or represents that its use would not infringe privately owned rights. Reference herein to any specific commercial product, process, or service by trade name, trademark, manufacturer, or otherwise does not necessarily constitute or imply its endorsement, recommendation, or favoring by the United States Government or any agency thereof. The views and opinions of authors expressed herein do not necessarily state or reflect those of the United States Government or any agency thereof. 


\section{DISCLAIMER}

Portions of this document may be illegible in electronic image products. Images are produced from the best available original document. 


\title{
Exotic Nuclei from a Theoretical Perspective
}

\author{
Witold Nazarewicz
}

\author{
Department of Physics, University of Tennessee, Knoxville, Tennessee $37996^{1}$ \\ Physics Division, Oak Ridge National Laboratory, Oak Ridge, Tennessee $37831^{2}$ \\ Institute of Theoretical Physics, University of Warsaw, ul. Hoza 69, PL-00-681 Warsaw, Poland
}

\begin{abstract}
One of the main frontiers of nuclear structure today is the physics of radioactive nuclear beams. Experiments with radioactive beams will make it possible to look closely into many aspects of the nuclear many-body problem. What makes this subject both exciting and difficult is: (i) the weak binding and corresponding closeness of the particle continuum, implying a large diffuseness of the nuclear surface and extreme spatial dimensions characterizing the outermost nucleons, and (ii) access to the exotic combinations of proton and neutron numbers which offer prospects for completely new structural phenomena.
\end{abstract}

\section{INTRODUCTION}

The field of radioactive nuclear beams (RNB) is one of the main frontiers of nuclear science today. One of the indications of the potential of this field is the large international interest in the development of facilities with RNB capabilities. At present there are only a few laboratories with radioactive ion beam capabilities. However, the prospects for new experiments and the success of the current programs have led to a number of RNB facilities under development, and a number of further proposals, including the construction of the next-generation facilities in Europe, U.S., and Japan.

Theoretically, exotic nuclei represent a formidable challenge for the nuclear manybody theories and their power to predict nuclear properties in nuclear "terra incognita". It is important to remember that the lesson learned by going to the limits of the nuclear binding is also important for "normal" nuclei from the neighborhood of the beta stability valley. And, of course, radioactive nuclei are crucial astrophysically; they pave the highway along which the nuclear material is transported up in the proton and neutron numbers during the complicated synthesis process in stars.

1) Research supported by the U.S. Department of Energy under Contract DE-FG02-96ER40963.

2) Research supported by the U.S. Department of Energy under Contract DE-AC05-96OR22464 with Lockheed Martin Energy Research Corp. 
Recent research relating to exotic nuclei has already demonstrated the potential for exciting new nuclear physics. Examples include the exotic structure of halo nuclei, which present us with new forms of nuclear matter, the surprising fragility of magic numbers, which hints at some of the marked changes in the underlying foundations of nuclear structure, and the production and study of the long-searched doubly closed shell nuclei ${ }^{78} \mathrm{Ni}$ and ${ }^{100} \mathrm{Sn}$. In the following, I shall briefly comment on some of the themes related to the RNB physics.

\section{THEORETICAL CHALLENGES FAR FROM STABILITY}

In the description of weakly bound systems, the major theoretical challenge is the correct treatment of the particle continuum. For weakly bound nuclei, the Fermi energy lies very close to zero, and the decay channels must be taken into account explicitly. As a result, many cherished approaches of nuclear theory must be modified. (For an extensive discussion of the theoretical perspectives far from stability, see the recent review [1].) But there is also a splendid opportunity: the explicit coupling between bound states and continuum, and the presence of lowlying scattering states invite strong interplay and cross-fertilization between nuclear structure and reaction theory.

How can one extend traditional tools of nuclear theory to account for the scattering of nucleons from bound single-particle orbitals to unbound states? The closeness of the particle continuum reverberates in two aspects of the theoretical description. Firstly, the particles forming a bound nuclear state can virtually scatter back and forth into the particle continuum phase space. This process must conserve the localization of the nuclear wave function which remains bound even with such a virtual scattering taken into account. A theoretical description of this kind of effect still remains virgin territory, although some progress has been made in the analysis of the virtual pair scattering $[2,3]$. Secondly, nucleons can very easily leave the nucleus altogether and enter the particle continuum through the real scattering. This is an old problem which, in the context of excited states near or above the particle threshold, has been addressed by the continuum shell model (CSM) [4-6]. In the CSM, the continuum states (decay channels) and bound states are treated on equal footing. Consequently, correlations due to the coupling to resonances, the spatial extension effects in weakly bound states, the structure of resonances, and the structure of particle transfer form factors are properly described by the CSM. Unfortunately, in many shell-model calculations for weakly bound nuclei (including these presented at this meeting!) the continuum aspects are completely disregarded; hence their conclusions should be taken with a grain of salt (see, however, the recent study [7]).

Often, particle continuum is approximated by the quasibound states, i.e., the states resulting from the diagonalization of a finite potential in a large basis [8,9] or by enclosing the finite nuclear potential within an infinite well with walls positioned 
at a large distance from the nuclear surface $[10,11]$. More sophisticated methods of discretizing continuum include the Sturmian function expansions [12-15] and resonant (Gamow) state expansions.

\section{APPLICATIONS OF GAMOW STATES TO WEAKLY BOUND NUCLEI}

The Gamow states are eigenstates of the time-independent Schrödinger equation with complex eigenvalues [16-18]. They are regular at $r=0$ and satisfy a purely outgoing wave type of asymptotics with the complex energy eigenvalue. The real part of the complex energy eigenvalue is the expectation value of the one-body Hamiltonian, while the imaginary part is related to the total decay width of the quasi-stationary state. The Gamow states are the poles of the $S$-matrix on the complex energy plane lying below the positive real axis. The closer they lie to the real axis, the more they resemble the bound states, and they can be associated with narrow resonances.

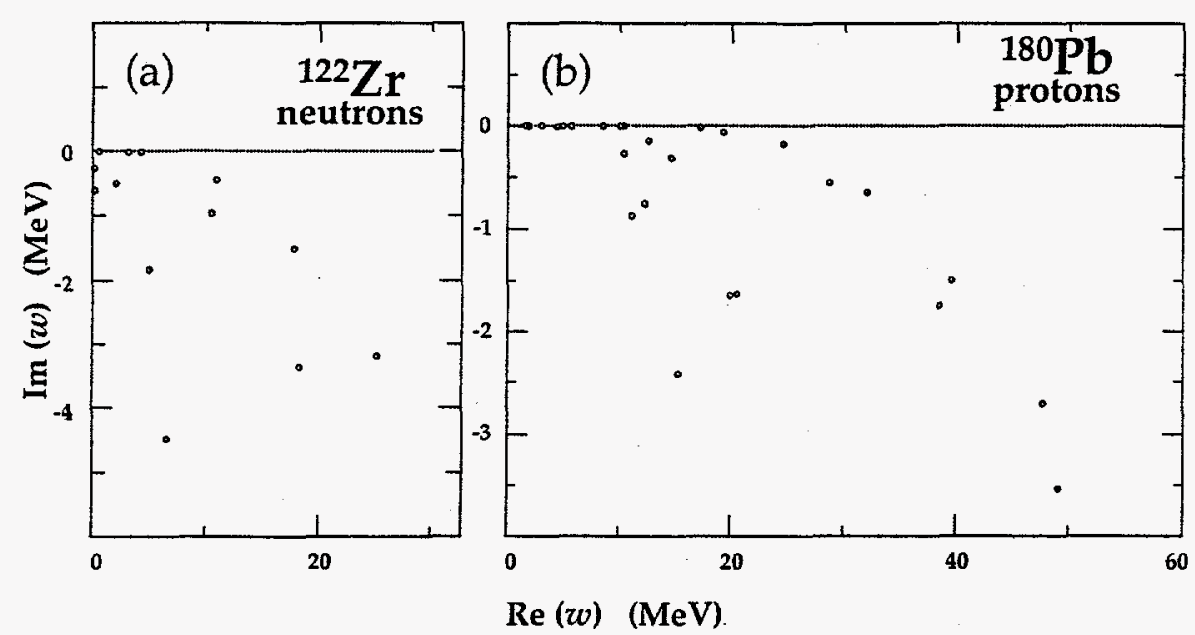

FIGURE 1. The distribution of Gamow energy eigenvalues $w_{i}$ in the $(\operatorname{Re}(w), \operatorname{Im}(w))$ plane for (a) neutron drip-line nucleus ${ }^{122} \mathrm{Zr}$ (neutron eigenvalues), and (b) proton-rich nucleus ${ }^{180} \mathrm{~Pb}$ (proton eigenvalues). (From Ref. [19].)

A generalized completeness relation proposed by Berggren [17] paved the way for using Gamow states as basis states in a similar way as the ordinary bound states are used. Using the generalized completeness relation, one can treat a selected set of resonant states on the same footing as bound states. The remaining part of the continuum is treated by means of the integral along a path in a complex energy plane. Recently, this technique has been applied to calculate the single-particle level density and shell corrections for finite depth potentials $[20,19]$. Figure 1 illustrates the distribution of spherical Gamow eigenvalues for ${ }^{122} \mathrm{Z}$ and ${ }^{180} \mathrm{~Pb}$ (all partial waves 
are shown). While for the neutrons in ${ }^{122} \mathrm{Zr}$ the large-width Gamow states appear just above the $\operatorname{Re}(w)=0$ threshold, for the protons in ${ }^{180} \mathrm{~Pb}$ the particle continuum is shifted effectively by $\sim 8 \mathrm{MeV}$ due to the presence of the Coulomb barrier. The proton Gamow states that appear at low energies are extremely narrow resonances, usually discussed in the context of proton emitters.

\section{MICROSCOPIC DESCRIPTION OF NUCLEAR MASSES}

In the description of weakly bound systems, pair scattering plays a unique role. In standard methods based on bound and quasibound states the virtual scattering of nucleonic pairs from bound states to the positive-energy states leads to the presence of a "particle gas" surrounding the nucleus [2]. This problem is overcome in the HFB method with a realistic pairing interaction in which the coupling of bound states to the particle continuum is correctly taken into account $[2,3,21,22]$. Consequently, for large exotic nuclei, the self-consistent HFB treatment is not a matter of choice, it is a must. The calculations are not easy, especially if the self-consistent symmetries (e.g., spherical symmetry) are broken.

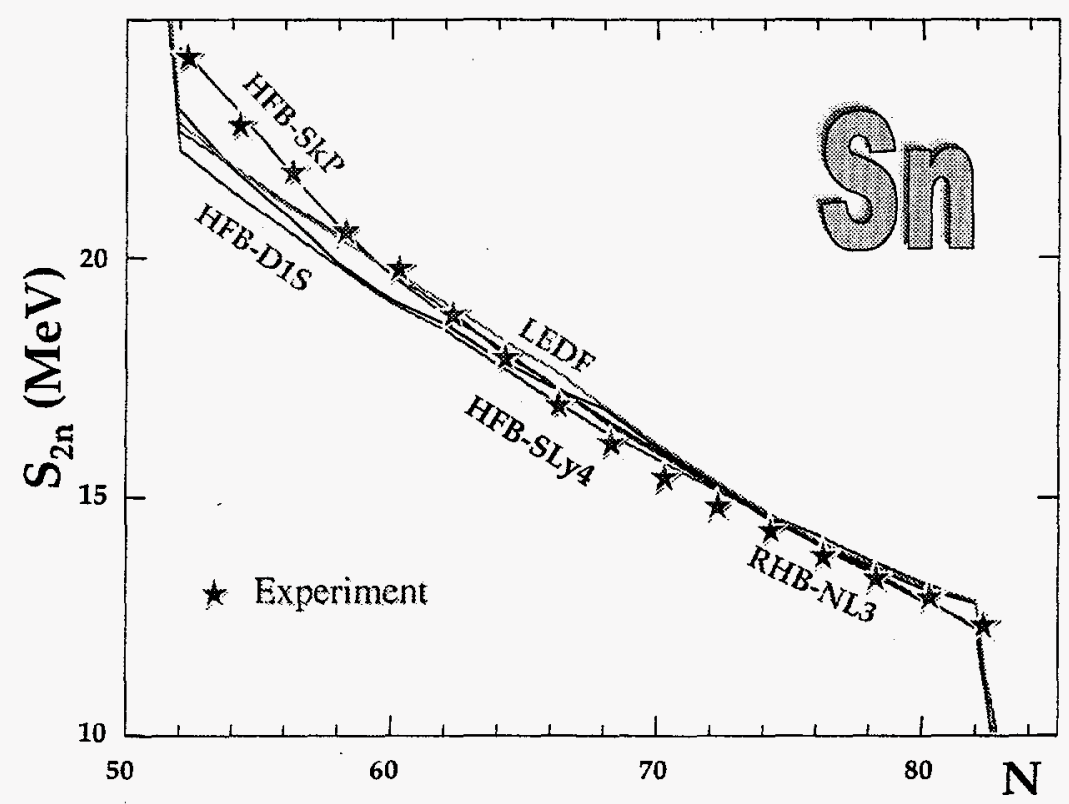

FIGURE 2. Two-neutron separation energies $S_{2 n}$ for the $\mathrm{Sn}$ isotopes calculated in five microscopic models: HFB-D1 (courtesy of Jacques Dechargé), HFB-SkP and HFB-SLy4 (courtesy of Jacek Dobaczewski), LEDF (courtesy of Sergei Fayans), and RHB-NL3 (courtesy of Georgios Lalazissis). The experimental data are indicated by stars.

For medium-mass and heavy nuclei, no-core microscopic-mass calculations (i.e., based on microscopic effective interactions and employing many-body theory) are 
usually performed within the mean-field approach. One has to bear in mind, however, that in the mean-field (i.e., independent-quasiparticle) theory, the correlations beyond pairing are not considered, and this gives rise to systematic deviations between experimental and calculated masses [23]. On the other hand, when comparing mass differences such as separation energies, the contributions originating from correlations beyond the mean field tend to cancel out. Figure 2 displays two-neutron separation energies for the $\mathrm{Sn}$ isotopes calculated in five state-of-the art microscopic models: HFB-D1S (based on finite-range Gogny interaction D1S [24]), HFB-SkP and HFB-SLy4 (based on zero-range Skyrme parametrizations SkP [2] and SLy4 [25]), LEDF (local energy-density functional model with parametrization FaNDF [26]), and RHB-NL3 (relativistic Hartree-Bogolyubov model with NL3 parametrization [27]). All models give a very good description of existing experimental data; some interesting deviations are seen when approaching the proton drip line.

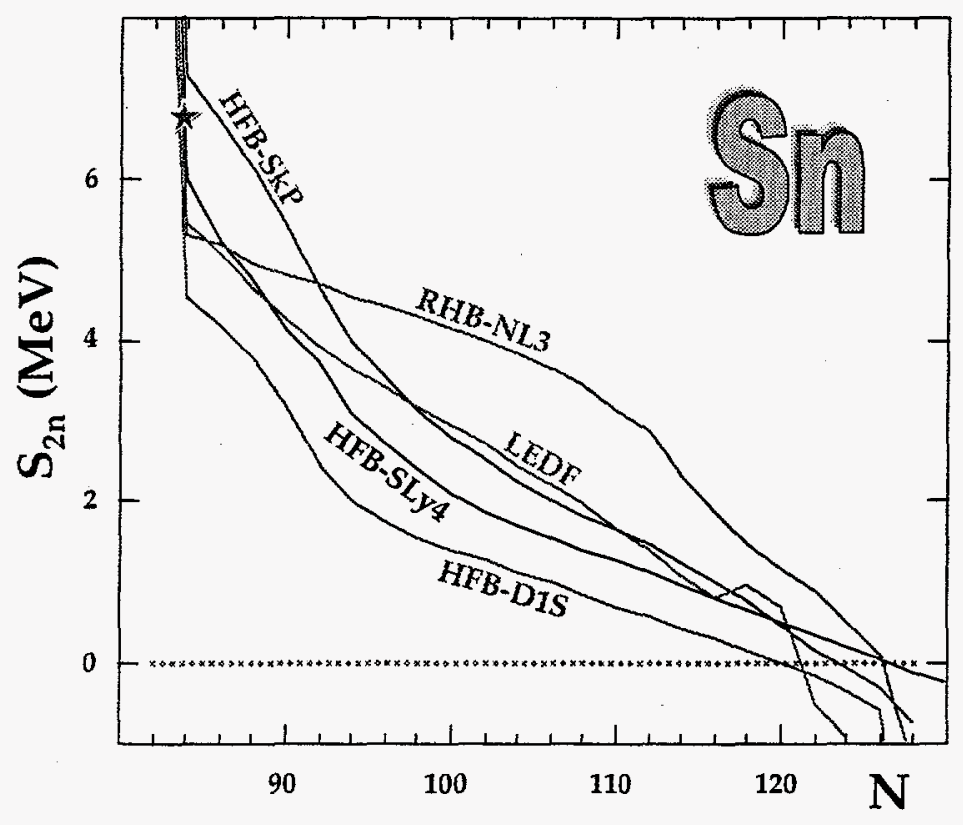

FIGURE 3. Similar to Fig. 2 except for very neutron-rich Sn isotopes.

How do all these microscopic models perform when extrapolated to exotic neutron-rich nuclei? This is presented in Fig. 3. Clearly, the differences between forces are greater in the region of "terra incognita" than in the region of known masses shown in Fig. 2. As seen in Fig. 3, the position of the neutron drip line for the $\mathrm{Sn}$ isotopes slightly depends on the effective interaction used; it varies between $N=120$ (HFB-D1S) and $N=126$ (RHB-NL3). Hence, even if the theoretical method used to calculate nuclear masses is reliable near the drip line, the uncertainty due to the largely unknown isospin dependence of the force gives an appreciable theoretical "error bar". A detailed analysis of the force dependence of results may give us valuable information on the relative importance of various force parameters and 
many-body approximations.

\section{ODD-EVEN STAGGERING OF NUCLEAR MASSES}

The presence of the odd-even staggering (OES) in nuclear binding energies [28] is usually attributed to the existence of nucleonic pairing correlations $[29,30]$. Recently, a similar effect has been observed for ultra-small superconducting metallic grains [31] and it is believed to result from the superconducting correlations [32]. Although the motion of electrons in metals is very different from that of nucleons in nuclei, the mechanism behind electronic and nucleonic superconductivity (the presence of an attractive residual interaction which gives rise to a correlated manyfermion system) is indeed very similar $[33,34]$. On the other hand, no evidence has been found for superconductivity in metal clusters, and the OES of binding energies in such systems is attributed to the Jahn-Teller effect which, by breaking the spherical symmetry of the mean field, gives rise to deformed single-particle orbitals $[35,36]$.

In a recent study [3T], the phenomenon of OES in nuclei has been analyzed using the self-consistent HF method. In the independent quasiparticle picture, the gap parameter is often related to the binding energies of three adjacent systems:

$$
\Delta^{(3)}(\mathcal{N}) \equiv \frac{(-1)^{\mathcal{N}}}{2}[B(\mathcal{N}-1)+B(\mathcal{N}+1)-2 B(\mathcal{N})]
$$

where $\mathcal{N}$ is the particle number (i.e, $N$ or $Z$ ). As shown in Ref. [37], the quantity $\Delta^{(3)}(n)$ calculated in the HF method without pairing is nearly zero for the odd values of $n$ and is positive for even values of $n$, reflecting the presence of the deformed mean field. This result suggests that both pairing and mean-field components of OES can be extracted from binding energies by using the three-point filter, $\Delta^{(3)}$. Namely, the values of $\Delta^{(3)}$ calculated at odd values of $n$ can be, roughly, associated with the pairing effect,

$$
\Delta_{\nu}(\mathcal{N}) \equiv \Delta_{\nu}^{(3)}(\mathcal{N}=2 n+1)
$$

while the differences of $\Delta^{(3)}$ at adjacent even and odd values of $n$ give information about the deformed single-particle spectra [37],

$$
\epsilon_{n+1}-\epsilon_{n}=2\left[\Delta_{\nu}^{(3)}(\mathcal{N}=2 n)-\Delta_{\nu}^{(3)}(\mathcal{N}=2 n+1)\right] .
$$

The neutron staggering parameter, $\Delta^{(3)}$, extracted from the experimental binding energies is shown in Fig. 4. The values of $\Delta_{\nu}^{(3)}$ at even neutron numbers are almost twice as large as those at odd neutron numbers. Therefore, in light nuclei the mean-field and pairing effects contribute almost equally to the staggering of nuclear masses. 


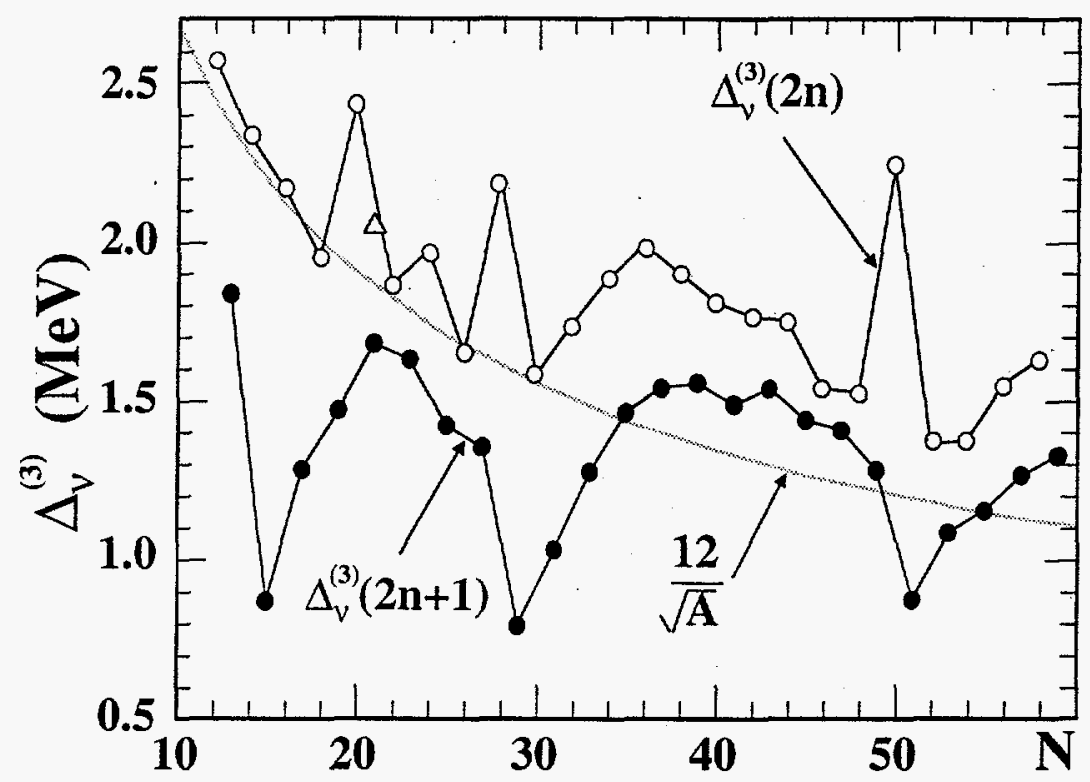

FIGURE 4. Experimental values of $\Delta_{\nu}^{(3)}(N)$. The thick gray line indicates the average trend, $\tilde{\Delta}=12 / \sqrt{A}$. Each point represents the arithmetic mean over several even- $Z$ isotones. (From Ref. [37].)

\section{SPECTROSCOPY OF PROTON EMITTERS}

Proton radioactivity is an excellent example of the elementary three-dimensional quantum-mechanical tunneling. Lifetimes of proton emitters directly provide an indication of the angular momentum content of the narrow proton resonance [38]. Experimental and theoretical investigations of proton emitters (or theoretically predicted ground-state di-proton emitters) are just opening up a wealth of exciting physics associated with the residual interaction coupling between bound states and extremely narrow resonances in the region of very low density of single-particle levels.

Recently, a method of calculating deformed proton resonances by means of the coupled-channel technique with Gamow states has been proposed $[39,40]$. In very deformed nuclei, proton resonances can be treated by means of the strong coupling approach. However, to investigate the influence of the angular momentum dependence of the proton decay width, this formalism has to be extended to account for the Coriolis coupling.

Another exciting avenue is the competition between gamma-radiation and the emission of prompt protons. Here, spectacular examples are proton-emitting intruder bands in ${ }^{58} \mathrm{Cu}[41]$ and ${ }^{56} \mathrm{Ni}[42]$. In ${ }^{56} \mathrm{Ni}$, where two intruder bands have been observed, the lower rotational band can be explained by large-scale shell-model calculations in the $p f$ shell. Also the results of cranked mean-field calculations in- 
dicate that this band is built upon a $4 \mathrm{p}-4 \mathrm{~h}$ excitation within the $p f$ shell (see Fig. $5,4^{0} 4^{0}$ band). The second band, however, is expected to involve particles in the $1 g_{9 / 2}$ orbit, which is supported by its nearly identical behavior to the band in ${ }^{58} \mathrm{Cu}$. However, the best scenario for this band is based on one proton promoted to the $1 g_{9 / 2}$ orbit $\left(4^{0} 4^{1}\right.$ band in Fig. 5), while a neutron and a proton occupies this orbit in ${ }^{58} \mathrm{Cu}$. The fact that our best theoretical scenario for deformed identical

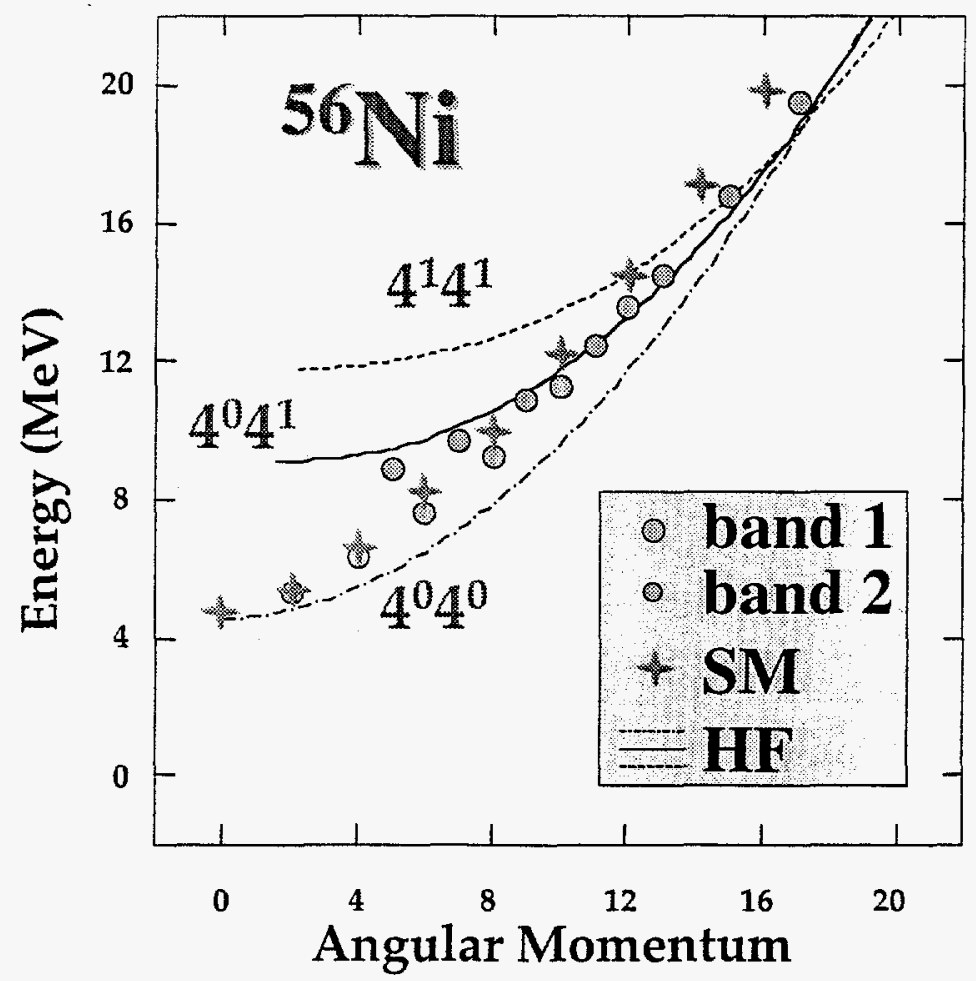

FIGURE 5. Excitation energy versus angular momentum for experimental and calculated intruder bands in ${ }^{56} \mathrm{Ni}$. Lines indicate the $4^{0} 4^{0}, 4^{0} 4^{1}$, and $4^{1} 4^{1}$ bands calculated in the cranked $\mathrm{HF}+\mathrm{SLy} 4$ model, while the KBF shell-model calculations (SM) are indicated by crosses.

bands in ${ }^{56} \mathrm{Ni}$ and ${ }^{58} \mathrm{Cu}$ involves structures with different intruder content is very puzzling and requires further investigations.

\section{CONCLUSIONS}

Studies of properties of nuclei far from the beta stability line, both the groundstate characteristics (masses, radii, deformations...) and the properties of excited states of exotic nuclei, are crucial for our understanding of the effective nucleonnucleon interaction and the behavior of the nuclear many-body system [43]. In this 
context, the study of matter with radioactive beams of nuclei is one of the most exciting challenges of nuclear physics and nuclear astrophysics today. The field is extremely rich and has a truly multidisciplinary character. Experiments with radioactive beams will make it possible to look closely into many exciting aspects of the nuclear many-body problem. Although an experimental excursion into new territories of the chart of the nuclides will offer many excellent opportunities for traditional nuclear structure, there are many unique features of exotic nuclei (associated with weak binding, large diffuseness, and large spatial dimensions) that give very good prospects for entirely new phenomena likely to be different from anything we have observed to date. A broad international community is enthusiastically using existing RNB facilities and hoping and planning for future-generation tools.

\section{REFERENCES}

1. J. Dobaczewski and W. Nazarewicz, Phil. Transactions, in press, 1998; nuclth/9707049.

2. J. Dobaczewski, H. Flocard, and J. Treiner, Nucl. Phys. A422, 103 (1984).

3. J. Dobaczewski, W. Nazarewicz, T.R. Werner, J.-F. Berger, C.R. Chinn, and J. Dechargé, Phys. Rev. C53, 2809 (1996).

4. U. Fano, Phys. Rev. 124, 1866 (1961).

5. C. Mahaux and H. Weidenmüller, Shell Model Approaches to Nuclear Reactions (North-Holland, Amsterdam, 1969).

6. R.J. Philpott, Fizika 9, suppl. 3, 21 (1977).

7. K. Bennaceur, F. Nowacki, J. Okołowicz, and M. Płoszajczak, J. Phys. G24, 1631 (1998).

8. M. Bolsterli, E.O. Fiset, J.R. Nix, and J.L. Norton, Phys. Rev. C5, 1050 (1972).

9. W. Nazarewicz, T.R. Werner, and J. Dobaczewski, Phys. Rev. C50, 2860 (1994).

10. J.R. Bennett, J. Engel, and S. Pittel, Phys. Lett. B368, 7 (1996).

11. F. Ghielmetti, G. Colo, E. Vigezzi, P.F. Bortignon, and R.A. Broglia, Phys. Rev. C54, R2143 (1996).

12. W. Glöckle, J. Hufner, and H.A. Weidenmüller, Nucl. Phys. A90, 481 (1967).

13. J.S. Vaagen, B.S. Nilsson, J. Bang, and R.M. Ibarra, Nucl. Phys. A319, 143 (1979).

14. G. Rawitscher, Phys. Rev. C25, 2196 (1982).

15. M. Buballa, S. Dróżdż, S. Frrewald, and J. Speth, Ann. Phys. 208, 346 (1991).

16. J. Humblet and L. Rosenfeld, Nucl. Phys. 26, 529 (1961).

17. T. Berggren, Nucl. Phys. A109, 265 (1968).

18. T. Vertse, P. Curutchet, and R.J. Liotta, Lecture Notes in Physics 325 (Springer Verlag, Berlin 1987), p. 179.

19. T. Vertse, R.J. Liotta, W. Nazarewicz, N. Sandulescu, and A.T. Kruppa, Phys. Rev. C57, 3089 (1998).

20. N. Sandulescu, O. Civitarese, R.J. Liotta, and T. Vertse, Phys. Rev. C55, 1250 (1997). 
21. S.T. Belyaev, A.V. Smirnov, S.V. Tolokonnikov, and S.A. Fayans, Sov. J. Nucl. Phys. 45, 783 (1987).

22. W. Poschl, D. Vretenar, and P. Ring, Comp. Phys. Commun. 103, 217 (1997).

23. Z. Patyk, A. Baran, J.F. Berger, J. Dechargé, J. Dobaczewski, R. Smolańczuk, and A. Sobiczewski, Acta Phys. Pol. B27, 457 (1996).

24. J. Dechargé and D. Gogny, Phys. Rev. C21, 1568 (1980).

25. E. Chabanat, Interactions effectives pour des conditions extrêmes d'isospin, Université Claude Bernard Lyon-1, Thesis 1995, LYCEN T 9501, unpublished.

26. S.A. Fayans, private communication; ENAM98, Abstracts, PB19, PB21.

27. G.A. Lalazissis, J. König, and P. Ring, Phys. Rev. C55, 540 (1997).

28. W. Heisenberg, Z. Phys. 78, 156 (1932).

29. A. Bohr, B.R. Mottelson, and D. Pines, Phys. Rev. 110, 936, (1958).

30. A. Bohr and B.R. Mottelson, Nuclear Structure, vol. 1 (W.A. Benjamin, New York, 1969).

31. C.T. Black, D.C. Ralph, and M. Tinkham, Phys. Rev. Lett. 76, 688 (1996).

32. R. Rossignoli, N. Canosa, and P. Ring, Phys. Rev. Lett. 80, 1853 (1998).

33. A. Bohr and B.R. Mottelson, Nuclear Structure, vol. 2 (W.A. Benjamin, New York, 1975).

34. H.J. Lipkin, APS News, January 1998.

35. K. Clemenger, Phys. Rev. B32, 1359 (1985).

36. M. Manninen, J. Mansikka-aho, H. Nishioka, and Y. Takahashi, Z. Phys. D31, 259 (1994).

37. W. Satuła, J. Dobaczewski, and W. Nazarewicz, Phys. Rev. Lett. (1998).

38. P.J. Woods and C.N. Davids, Ann. Rev. Nucl. Part. Sci. 47, 541 (1997).

39. E. Maglione, L.S. Ferreira, and R.J. Liotta, Phys. Rev. Lett. 81, 538 (1998).

40. A. Kruppa et al., in preparation.

41. D. Rudolph, C. Baktash, J. Dobaczewski, W. Nazarewicz, W. Satuła, M.J. Brinkman, M. Devlin, H.-Q. Jin, D.R. LaFosse, L.L. Riedinger, D.G. Sarantites, and C.-H. Yu, Phys. Rev. Lett. 80, 3018 (1998).

42. D. Rudolph, C. Baktash, M.J. Brinkman, E. Caurier, D.J. Dean, M. Devlin, J. Dobaczewski, P.-H. Heenen, H.-Q. Jin, D.R. LaFosse, W. Nazarewicz, F. Nowacki, A. Poves, L.L. Riédinger, D.G. Sarantites, W. Satula, and C.-H. Yu, submitted to Phys. Rev. Lett.

43. Scientific Opportunities With an Advanced ISOL Facility, Report, November 1997. 